\title{
Scattered and Fluorescent Photon Track Reconstruction in a Biological Tissue
}

\author{
Maria N. Kholodtsova, ${ }^{1,2,3}$ Pavel V. Grachev, ${ }^{1}$ Tatiana A. Savelieva, ${ }^{1}$ Nina A. Kalyagina, ${ }^{1,2,3}$ \\ Walter Blondel, ${ }^{2,3}$ and Viktor B. Loschenov ${ }^{1}$
}

${ }^{1}$ A. M. Prokhorov General Physics Institute of the Russian Academy of Sciences, Vavilov Street 38, Moscow 119991, Russia

${ }^{2}$ Université de Lorraine, CRAN, UMR 7039, 2 avenue de la Forêt de Haye, 54516 Vandoeuvre-Lès-Nancy Cedex, France

${ }^{3}$ CNRS, CRAN, UMR 7039, 2 avenue de la Forêt de Haye, 54516 Vandoeuvre-Lès-Nancy Cedex, France

Correspondence should be addressed to Maria N. Kholodtsova; m.kholodtsova@gmail.com

Received 6 November 2013; Revised 13 February 2014; Accepted 15 February 2014; Published 25 March 2014

Academic Editor: Alexandre Douplik

Copyright (C) 2014 Maria N. Kholodtsova et al. This is an open access article distributed under the Creative Commons Attribution License, which permits unrestricted use, distribution, and reproduction in any medium, provided the original work is properly cited.

\begin{abstract}
Appropriate analysis of biological tissue deep regions is important for tumor targeting. This paper is concentrated on photons' paths analysis in such biotissue as brain, because optical probing depth of fluorescent and excitation radiation differs. A method for photon track reconstruction was developed. Images were captured focusing on the transparent wall close and parallel to the source fibres, placed in brain tissue phantoms. The images were processed to reconstruct the photons most probable paths between two fibres. Results were compared with Monte Carlo simulations and diffusion approximation of the radiative transfer equation. It was shown that the excitation radiation optical probing depth is twice more than for the fluorescent photons. The way of fluorescent radiation spreading was discussed. Because of fluorescent and excitation radiation spreads in different ways, and the effective anisotropy factor, $g_{\text {eff }}$, was proposed for fluorescent radiation. For the brain tissue phantoms it were found to be $0.62 \pm 0.05$ and $0.66 \pm 0.05$ for the irradiation wavelengths $532 \mathrm{~nm}$ and $632.8 \mathrm{~nm}$, respectively. These calculations give more accurate information about the tumor location in biotissue. Reconstruction of photon paths allows fluorescent and excitation probing depths determination. The $g_{\text {eff }}$ can be used as simplified parameter for calculations of fluorescence probing depth.
\end{abstract}

\section{Introduction}

Methods of fluorescence diagnosis (FD) and photodynamic therapy (PDT) are widely used in almost all medical fields, that is, dermatology, ophthalmology, gynecology, and urology, as well as in neurology [1-4]. Intraoperative FD in neurology is a rapidly developing area. It allows differentiation of altered brain tissue from normal brain tissue with high specificity and sensitivity and as consequence helps to prevent resection of healthy tissue $[5,6]$. However, there are limits to the applicability of the method to the spinal cord and brain surgical interventions, which needs to be further addressed.

One of the most widely used photosensitisers for the FD for brain tissue is 5-aminolevulinic acid (5-ALA) which induces elevated protoporphyrin IX (PpIX) production in malignant cells. The PpIX fluoresces in the $630-700 \mathrm{~nm}$ wavelength range and is excited throughout the entire optical region. However, the wavelengths of 405,532 , and $632.8 \mathrm{~nm}$ are usually used as excitation wavelengths during laserinduced FD $[6,7]$. The long wavelength irradiation is of special interest due to its deep penetration into biological tissue, whereas the short-wavelength irradiation can provide higher resolution. In this work the investigation was conducted in two regions, the red $(\lambda=632.8 \mathrm{~nm})$ and green $(\lambda=532 \mathrm{~nm})$ regions of the spectrum.

By means of fluorescent diagnosis surgeon decides to reject or to leave tissue, so it is necessary to know from which area or depth all the data were collected to leave the healthy tissue and to remove tumor which accumulate photosensitiser.

The aim of the work was the estimation of the optical probing depth dependence on the distance between source and detector, allowing for an interpretation of FD results. We describe an experiment carried out on brain tissue phantoms, 


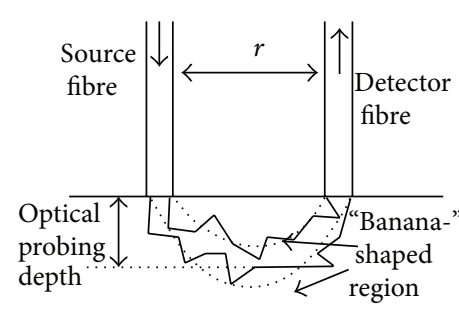

(a)

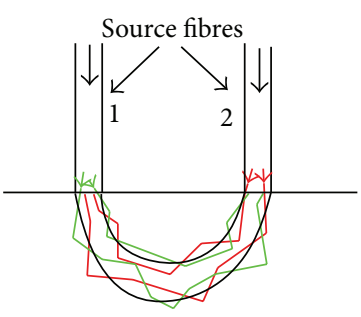

(b)

FIgURE 1: The most probable photon path distribution for (a) photons migrating from the source fibre to detector fibre, where the most probable area is limited by a "banana-" shaped region (dotted line) and (b) for photons migrating from two source fibres.

then compare it with numerical calculations using Monte Carlo (MC) modeling, and describe the use of self-developed software and theoretical results, based on the diffusion theory (DT) for analysis of photon migration in biological and other turbid media. It should be taken into account that the results of the experiment on the phantoms highly depend on the configuration of the fibres such as numerical aperture and diameter.

During surgery, it is important to remove a tumor without damaging healthy tissue. It is also essential to develop diagnostic methods, allowing for precise determination of the depth about which information can be collected during FD. We propose the effective fluorescence $g$-factor to be a parameter as it can show the depth of fluorescent photons propagation after their emission due to excitation of a photosensitiser contained in the tissue.

\section{Material and Methods}

2.1. Diffusion Theory of the Radiative Transfer Equation. According to the DT the incident photons in a semi-infinite medium will migrate to the fibre detector through the "banana-" shaped area. The ends of such a region originate from the fibres, and the middle reaches the deepest part [8]. Such a form is derived from the analytical DT solution of the radiative transfer equation (RTE) $[9,10]$. The radiation distribution equation at point $\overrightarrow{r_{1}}=(x, y, z)$ is as follows [10]:

$$
P(x, y, z)=\frac{z^{2} e^{-k\left(\sqrt{(r-x)^{2}+y^{2}+z^{2}}+\sqrt{\left.x^{2}+y^{2}+z^{2}\right)}\right.}\left(k \sqrt{(r-x)^{2}+y^{2}+z^{2}}+1\right)\left(k \sqrt{x^{2}+y^{2}+z^{2}}+1\right)}{\left((r-x)^{2}+y^{2}+z^{2}\right)^{3 / 2}\left(x^{2}+y^{2}+z^{2}\right)^{3 / 2}},
$$

with $r$-distance between two fibres, $L_{a}$-absorbance length (in $\mathrm{cm}$ ) where $L_{a}=\sqrt{D / \mu_{a}}$ and $k=1 / L_{a}, D$-diffusion coefficient where $D=1 /\left(3\left[\mu_{a}+(1-g) \mu_{s}\right]\right), \mu_{a}$-absorbance coefficient (in cm ${ }^{-1}$ ), $\mu_{s}$-scattering coefficient (in $\mathrm{cm}^{-1}$ ), and $g$-mean scattering cosine.

Given that $y=0$ (because of symmetry) and due to weak absorption $(k r \ll 1)$ it can be pointed out that the minima of the photon distribution function $P(x, y, z)$ in $Y Z$-plane is as follows [10]:

$$
\begin{aligned}
& z(r) \\
& =\sqrt{\frac{1}{8}\left[\sqrt{\left(x^{2}+(r-x)^{2}\right)^{2}+32 x^{2}(r-x)^{2}}-x^{2}-(r-x)^{2}\right]} .
\end{aligned}
$$

Hence the optical probing depth is defined as the maximum distance from an irradiated object surface between source and detector to the most probable photon migration area (Figure 1) whereby (2) defines the optical probing depth for a fixed distance between source and detector.

In this contribution, the equations above were used because we are not considering the signal on the surface which is detected by fibres, but we are investigating the path of photons propagating in the medium.

2.2. Monte Carlo Simulations. MC modeling is well known and widely used for biological tissue optical properties derivation [10, 11]. This method is based on numerical modeling of photon propagation in turbid media. During migration, photons undergo multiple scattering processes and, finally, absorption.

We developed the MClight program which models threedimensional (3D) light distribution in turbid media. One feature of this program is the ability to set different geometries for the experiment such as fibre position, numerical aperture (NA), and diameter of fibres, as well as the multilayered model where one can set optical properties $\left(\mu_{a}, \mu_{s}, g\right)$ and the thickness of each layer. The results of calculations are presented as a black and white intensity distribution image. Such a representation is clear and allows comparison with the experimental data. Each pixel of this $\mathrm{b} / \mathrm{w}$ image corresponds to a point in the medium: if a photon is scattered at this point, the brightness at this point will be increased by one. As more photons are scattered at this point, the brighter the pixel will be. Resolution was $5 \mu \mathrm{m}$ per pixel as it was sufficient to 
be compared with the experimental data, also the resolution could be changed from $2 \mu \mathrm{m} / \mathrm{p}$ to $20 \mu \mathrm{m} / \mathrm{p}$.

It should also be taken into account that the image of 32 bit color depth allows small brightness changes in a light field to be detected, so the derived distributions are saved with *.tif extension. Another self-developed program (TiffReader) allows processing files with ${ }^{*}$.tif extension (if the color depth is more than 32 bytes, then the image is processed without image quality loss). This program has the ability to measure the distance between two chosen points, thereby obtaining brightness profiles as well as $3 \mathrm{D}$ brightness distribution. It is also possible to reduce noise by means of Sobel and Prewitt masks [12].

Moreover, the program includes maximum brightness and optical probing depth search algorithms. The algorithm is as follows: the area of the medium which photons cross the most is the brightest. According to this, the program searches for the brightest points and derives the area, where the photon flow is at its highest level. This area is colored red and the results can be saved in a graphics file. The distance between the surface plane and the lowest point of the obtained region are the optical probing depth, which can be measured 4 directly in the program developed.

2.3. Determination of Anisotropy Factor. Firstly, brain tissue phantoms were made for comparative pictures of photon distribution. These were made up of "Intralipid 10\%" and sulfonated aluminum phthalocyanine (Photosens, Science Center "NIOPIK", Moscow, Russia) at a concentration of $2 \mathrm{mg} / \mathrm{kg}$ (recommended dosage for FD) and had similar scattering and absorbing properties to real brain tissue. As "Intralipid 10\%" is not an optical standard, it has a bottleto-bottle variation in the optical properties, which had to be verified experimentally for each bottle at the time of use $[13,14]$. As the main parameter affecting the photon distribution in a single scattering medium is the anisotropy factor ( $g$-factor), the scattering $\left(\mu_{s}\right)$ and absorption $\left(\mu_{a}\right)$ coefficients are scaling and have an influence on photon free path length. Consequently, in order to obtain the $g$-factor, goniophotometry was used in a spectrometer of dynamic light scattering (Photocor Complex optical unit; Photocor Instruments, Inc., College Park, MD, USA) (Figure 2).

To perform this method, the layer of Intralipid (1.6\% in water solution, $70 \mu \mathrm{m}$ thick) was placed in a special cuvette with $150 \mu \mathrm{m}$ thick walls and the indicatrix was obtained by means of photon counter (Photocor-PC2; Photocor Instruments, Inc., College Park, MD, USA). The results were approximated with the Henyey-Greenstein (HG) phase function, using the least square method. Also the free path length of photon in medium was determined, which is defined as a reversed sum of scattering and absorption coefficients.

2.4. Experimental Setup. A special methodology of the experiment was developed to determine the optical probing depth taking the following into account. As induced photons from one fibre migrate to another through the "banana-" shaped region (by virtue of the symmetry and isotropy of the turbid medium), changing the detector fibre to the source fibre

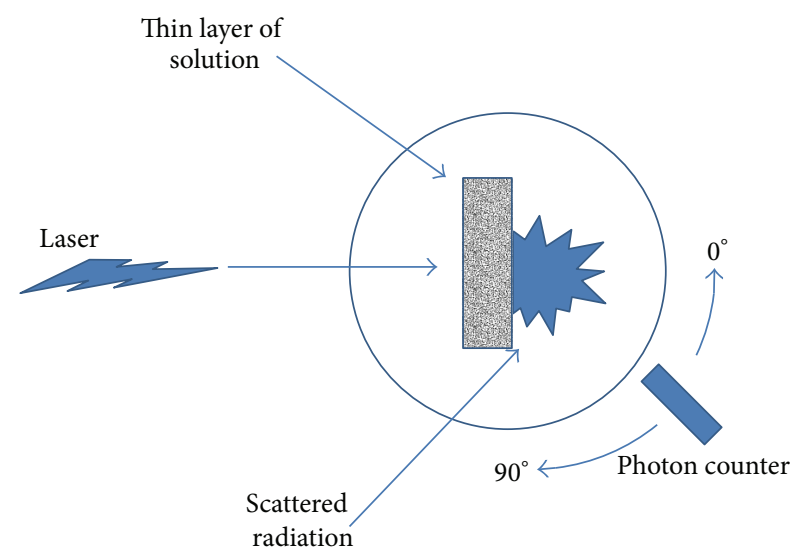

FIGURE 2: Scattering indicatrix measurement setup. Laser wavelength is $632.8 \mathrm{~nm}$.

will not affect the photon distribution because a correlation between the two fibres is absent. Also the areas of the most probable photon migration paths, from one fibre to another, add up (Figure 1(b)). Thus, using the TiffReader program, it is possible to obtain both regions where photons migrate through the paths distributed in a "banana-" shaped region and the optical probing depth.

The experiments were performed in four steps.

Firstly, in order to obtain the light field of diffusely scattered photon distribution between two fibres a setup was designed, which can be described as follows. It consists of a special cuvette $(50 \mathrm{~mm} \times 60 \mathrm{~mm} \times 30 \mathrm{~mm})$ with a thin transparent wall $(80 \mu \mathrm{m})$, optical fibres (material: quartzpolymer; NA $=0.48$; diameter $=600 \mu \mathrm{m}$ ), a b/w 32 bit color depth camera (VideoScan-415; VideoScan, Moscow, Russia), and two lasers, that is, a helium neon $(\mathrm{HeNe})$ laser $(\lambda=$ $632.8 \mathrm{~nm}, P=150 \mathrm{~mW}$; Biospec, Moscow, Russia) and a continuous diode $(\lambda=532 \mathrm{~nm}, P=150 \mathrm{~mW}$; Biospec, Moscow, Russia). The fibres were placed in the cuvette at a depth of $10 \mathrm{~mm}$ and close to the transparent wall. Then the images of photon path distribution were recorded with the camera which was placed parallel to the transparent wall. As the distortions obtained are minimal (due to thin wall presence), the effects of multipath are also minimal. This is because the photon path length is compared with the wall thickness and the refractive index of the wall differs from that of the solution by no more than $10 \%$. Furthermore, the photon path distribution is parallel to the wall and symmetrical to the plane which goes through the middle of the fibres.

Secondly, to obtain the fluorescent light field distribution, optical filters were used (for $532 \mathrm{~nm}$ light excitation: Orange Glass-14 ( $\left.T_{50} \approx 580 \mathrm{~nm}, T_{100} \approx 620 \mathrm{~nm}\right)$, for $632.8 \mathrm{~nm}$ : Red Glass-17 $\left(T_{50} \approx 670 \mathrm{~nm}, T_{100} \approx 700 \mathrm{~nm}\right)$ ). These filters were placed between VideoScan camera and the transparent wall of the cuvette. In order to reduce the signal-to-noise ratio, 10 images of each field distribution were averaged. During the experiment, the distance between the sources was varied in the range $0.5-7.5 \mathrm{~mm}$ (Figure 3). 


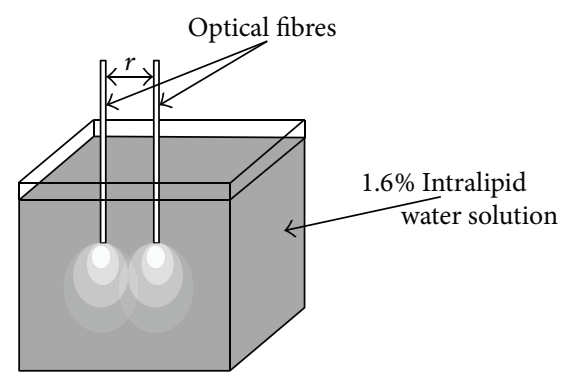

FIGURE 3: Scheme of the experimental setup.

TABLE 1: Optical properties of the brain tissue model for Photosens emission wavelength $(668 \mathrm{~nm})[15,16]$.

\begin{tabular}{lccc}
\hline Material & $\mu_{s}\left(\mathrm{~cm}^{-1}\right)$ & $\mu_{a}\left(\mathrm{~cm}^{-1}\right)$ & $g^{*}$ \\
\hline $1.6 \%$ Intralipid/water solution & $\sim 274$ & $\sim 0.001$ & $\sim 0.71$
\end{tabular}

${ }^{*}$ Not used in the calculations.

Thirdly, the 3D light distributions were obtained by means of MClight program for the two excitation wavelengths $(532 \mathrm{~nm}$ and $632.8 \mathrm{~nm}$ ). The geometry of the simulations replicates that of the real experiment and the optical properties of the simulated turbid medium were chosen according to the optical properties of Intralipid at the already mentioned wavelengths.

Finally, fluorescence and excitation field distributions obtained experimentally were processed in TiffReader using MClight after which the optical probing depths and regions of the most probable photon path were obtained. The optical probing depth was the distance from the end of fibre till the middle of the fluorescent or excitation field.

2.5. Effective Anisotropy Factor of Fluorescence. It was assumed that the effective fluorescent $g$-factor depends on several events such as scattering emitted from the source photons in tissue, fluorescent photon emission in any direction with equal probability, scattering of photon with the wavelength corresponding to the fluorescent wavelength of photosensitiser in tissue, and, finally, absorption of this photon or its escape from the area under consideration. So for the brain tissue phantoms containing the photosensitiser Photosens, the fluorescent effective $g$-factors were derived using four parameters. These were the experimentally derived optical probing depth, the preset distance between fibres (not shown in Table 1), and the known optical properties (scattering and absorption coefficient) of the model at the photosensitiser emission wavelength $\lambda=668 \mathrm{~nm}$ (Table 1 ). These parameters were substituted in (1) and then the effective anisotropy factor of fluorescence was derived using the mean root square method.

\section{Results and Discussion}

3.1. Determination of Anisotropy Factor. The anisotropy factor for $\lambda=632.8 \mathrm{~nm}$ was found to be $0.83 \pm 0.03$, the free path length $27 \pm 2 \mu \mathrm{m}$, and for $\lambda=532 \mathrm{~nm} 0.85 \pm 0.03$ and $20 \pm 3 \mu \mathrm{m}$,

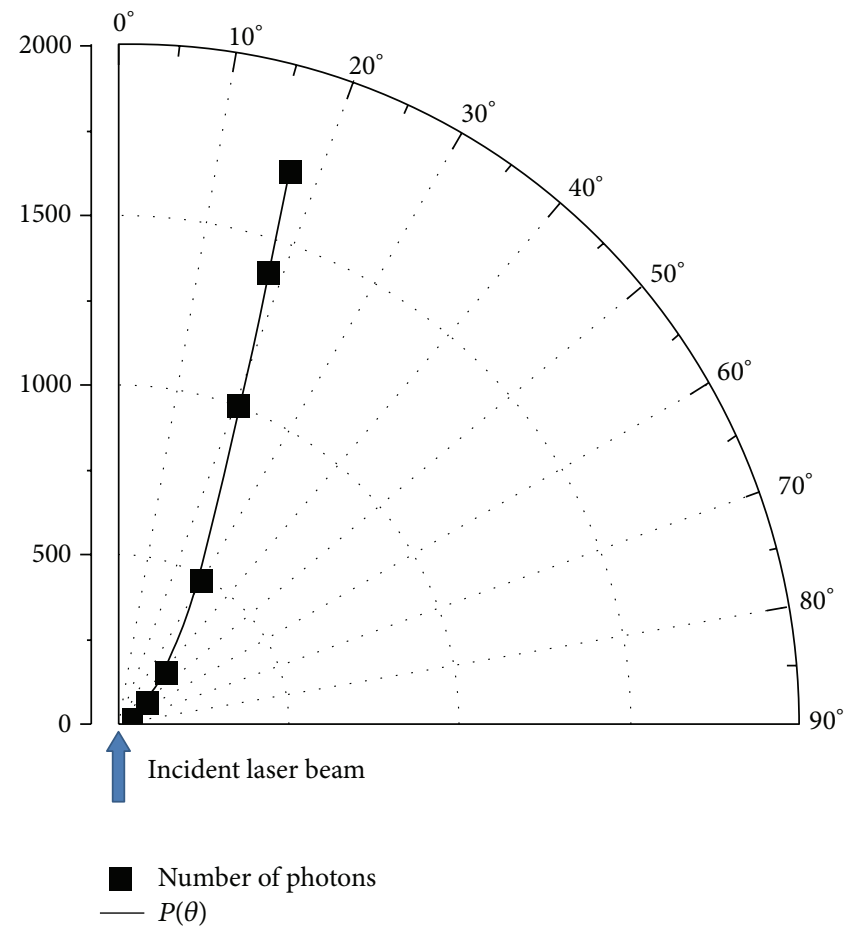

Figure 4: Dependence of the photon number counter on angle $\theta$. Experimental results are marked with squares; line is HG function for $g=0.83$.

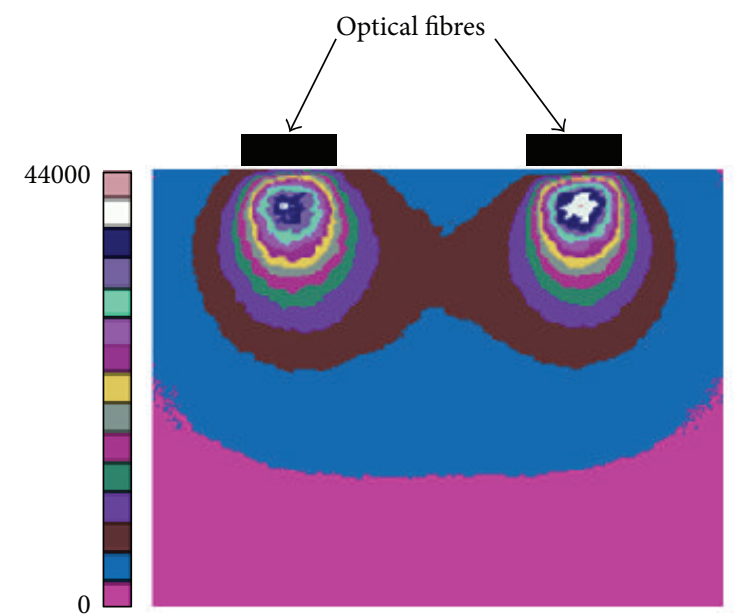

FIGURE 5: The image of light field distribution obtained by MClight and processed by means of the TiffReader program. Each tone corresponds to its own brightness range which is shown on the left side.

respectively, (Figure 4). These results are acceptable and can be used to model brain tissue and as input parameters for MC simulations.

3.2. Monte Carlo Simulations and the Diffusion Theory of the Radiative Transfer Equation. Firstly, the light field distribution was obtained by MClight and processed in TiffReader program (Figure 5). In this distribution, each color corresponds to its own brightness range. One can see that the 


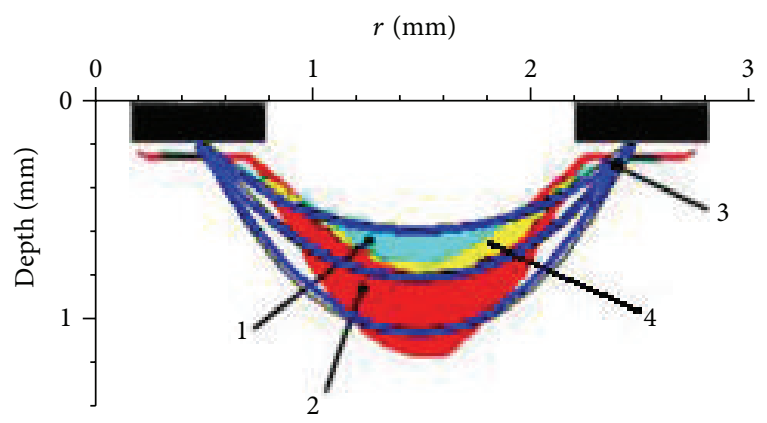

(a)

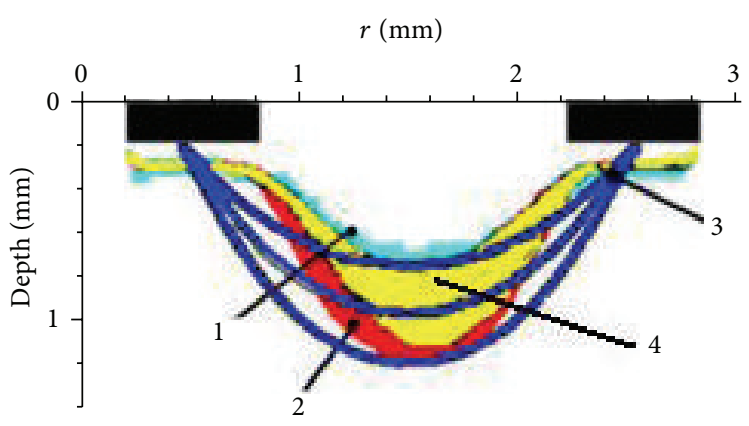

(b)

Figure 6: The most probable photon paths distribution between two sources for (a) $532 \mathrm{~nm}$ and (b) $632.8 \mathrm{~nm}$ (distance between the fibres, $1.7 \mathrm{~mm}$ ). 1-Experiment, 2-MC simulations, 3-diffuse approximation, and 4-intersection of 1 and 2.

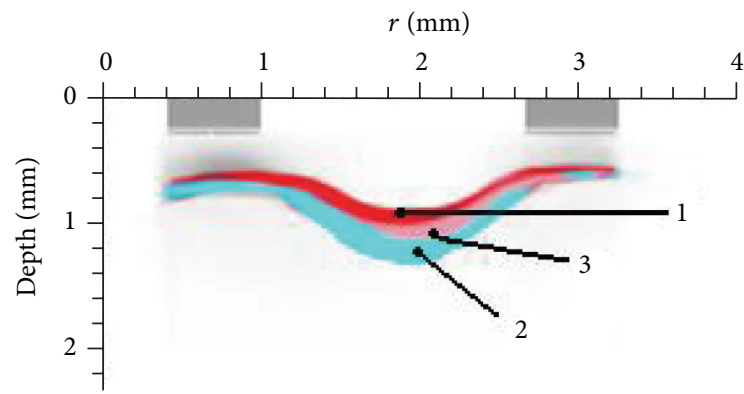

FIGURE 7: The most probable path distribution between two sources for fluorescent and diffusely scattered photons (excitation wavelength, $532 \mathrm{~nm}$; distance between fibres, $1.7 \mathrm{~mm}$ ). 1-fluorescent photons, 2-diffusely scattered photons, and 3-intersection of 1 and 2 .

maximum brightness is $300 \mu \mathrm{m}$ deeper than the ends of the fibre tips because in these areas brightness is the sum of the diffusely scattered light and the light emitted from fibres, without interaction with medium.

Secondly, the theoretical MC simulations were compared with the experimental results for the most probable photon propagation paths. These distributions were derived for two wavelengths of excitation radiation. Theoretical borders for the "banana-" shaped region were derived from (2). These areas are shown in Figure 6.

It is apparent that nearby the fibres' tips, the obtained theoretical results do not fit the experimental one. This fact is explained by the point-source assumption of the DT.

Thirdly, the experimentally obtained distributions of the most probable paths of fluorescent and excitation photons paths distributions are compared in Figure 7.

After that, the dependencies of the optical probing depth on the distance between the fibres for fluorescent and diffusely scattered light were determined. These results are presented in Figure 8. From the data in Figure 8 it can be seen that the optical probing depth dependencies on the distance between the fibres match the theoretical models as well as the experimental data (coefficients of determination $R^{2}$ are shown near corresponding lines). It was also found that the optical probing depth for an excitation wavelength of $532 \mathrm{~nm}$

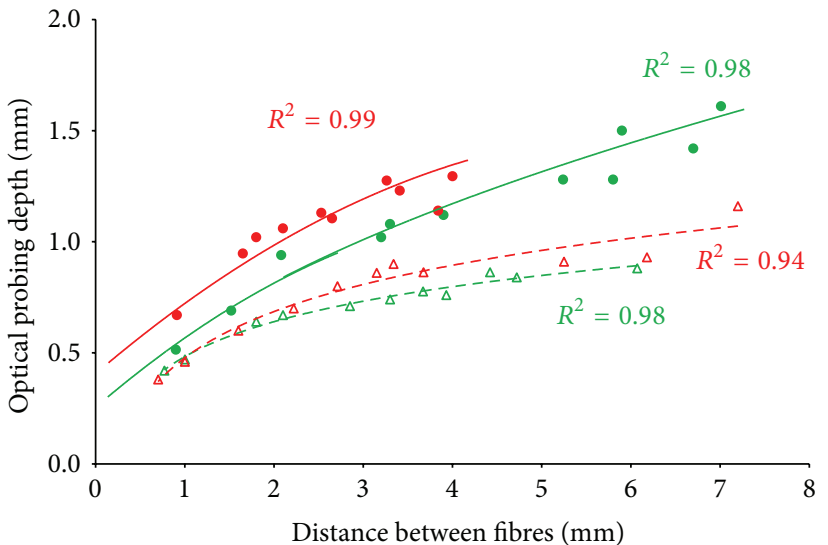

FIGURE 8: Dependence of optical probing depth on the distance between the fibres. Green: excitation wavelength $\lambda=$ $532 \mathrm{~nm}$ (circles-diffusely scattered radiation, full line-solution of (2), triangles-fluorescent radiation, and dashed line-approximation with logarithmic function). Red: excitation wavelength $\lambda=632.8 \mathrm{~nm}$ (circles-diffusely scattered radiation, full linesolution of (2), triangles-fluorescent radiation, and dashed lineapproximation with logarithmic function).

TABLE 2: Optical properties of the brain tissue model $[15,16]$.

\begin{tabular}{lcccc}
\hline Material & Wavelength $(\mathrm{nm})$ & $\mu_{s}\left(\mathrm{~cm}^{-1}\right)$ & $\mu_{a}\left(\mathrm{~cm}^{-1}\right)$ & $g$ \\
\hline 1.6\% Intralipid/ & 532.0 & 514 & 0.008 & 0.85 \\
water solution & 632.8 & 341 & 0.003 & 0.83 \\
Brain tissue & 532.0 & 410 & 0.01 & 0.87 \\
(white matter) & 632.8 & 409 & 0.08 & 0.84 \\
\hline
\end{tabular}

is less than for $632.8 \mathrm{~nm}$ on $20-30 \%$. This fact is explained by the difference in scattering coefficients, which are being lower for the shorter wavelength (Table 2).

Moreover, it was noticed that the optical probing depth dependence on the distance between the fibres for fluorescent light is approximated with the logarithmic function $a \ln (r)+$ $b$, where $r$ is the distance between the fibres and $a, b$ are the proportionality factors. $R^{2}$ is 0.95 and 0.98 for $632.8 \mathrm{~nm}$ and for $532 \mathrm{~nm}$, respectively (shown in Figure 8). 


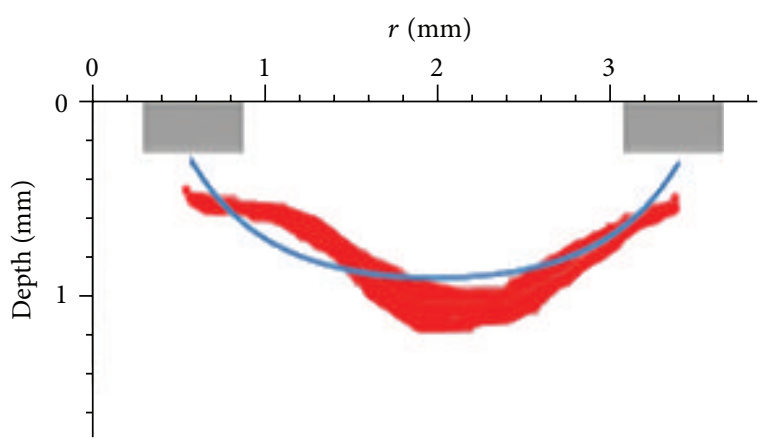

(a)

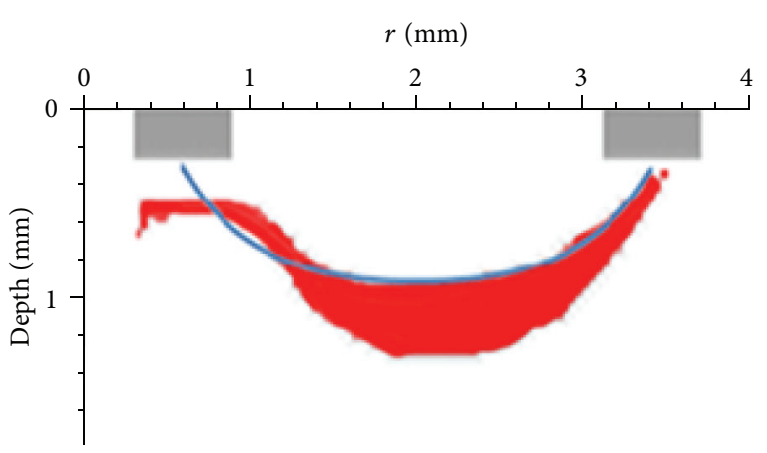

(b)

Figure 9: Calculated effective anisotropy factor substituted in (1) and experimental most probable photon path for (a) $532 \mathrm{~nm}$ excitation and (b) $632.8 \mathrm{~nm}$ excitation. Distance between fibres is $2.2 \mathrm{~mm}$.

TABLE 3: Effective fluorescent $g$-factor for the two-excitation wavelengths.

\begin{tabular}{lcc}
\hline Excitation wavelength $(\mathrm{nm})$ & 532.0 & 632.8 \\
\hline Effective $g$-factor & $0.62 \pm 0.05$ & $0.66 \pm 0.06$ \\
\hline
\end{tabular}

It is also important that the fluorescent probing depth is less than the excitation depth by $60-70 \%$ (it is followed from calculations and can be seen from Figure 8).

3.3. Effective Fluorescent g-Factor. After derivation of the probing depth for fluorescence, it was then possible to determine the effective fluorescent $g$-factor; the results are presented in Table 3 and Figure 9.

The error which can occur if one uses an ordinary $g$-factor for fluorescence radiation while determining the optical probing depth was calculated. In the case of $532 \mathrm{~nm}$ excitation, the error can be estimated to be $14 \%$, and in the case of $632.8 \mathrm{~nm}$, the error is 7\%. So it can be concluded that use of the effective fluorescent $g$-factor gives more accurate results and that the error will be higher for the shorter irradiation wavelength.

3.4. Discussion. The theoretical study of photon migration in a tissue has been performed by several research groups $[8,9,11]$. They considered the DT of the RTE and used MC simulations for light propagation in a turbid medium, including experiments on biological tissue phantoms. Equations for photon path distribution in presence and absence of absorbing element between emitter and detector were also derived. The authors showed that the photon flow through medium is in a confined region and suggested that distribution of photon paths may affect the spatial resolution.

We performed the calculations of photon migration process in a brain tissue phantom comparable with white matter from the brainas this is where malignant tumors mostly occur, using the excitation wavelengths most commonly used for FD during surgical interventions. It should be mentioned that if measurements are made on real brain tissue, the error will be greater for green light than for the red (absorbance of hemoglobin is 50x more for $532 \mathrm{~nm}$ than for $632.8 \mathrm{~nm}$ ). This is because the given results were obtained without taking the hemoglobin absorbance into consideration as soon as hemoglobin content highly depends on physiological state of tissue and requires deeper investigation.

The optical probing depth for shorter wavelength excitation is less than that for the longer ones. This can be explained by the difference in mean free path length; for shorter wavelengths it is lower so light is scattered earlier, and because of the lower $g$-factor, light is scattered less in a forward direction. Thus, the optical probing depth of $632.8 \mathrm{~nm}$ excitation is higher for $20-30 \%$ than for $532 \mathrm{~nm}$.

Considering the fluorescent optical probing depth, it is lower for shorter excitation wavelengths. This difference can be explained by several factors. First of all, it is partly due to the NA of the fibre which affects the initial angle of the photons emitted from the fibre. Secondly, the following motion of photons is defined by a $g$-factor, the closer it is to 1 , and the less the photons are deviated from the initial direction. Thus, the "banana-" shaped region is the result of step-by-step deviation of the photons from the initial direction. The absence of a propagation direction is the distinctive feature of fluorescent photons relative to diffusely scattered ones. A fluorescent photon is emitted by an excited atom in a $4 \pi$ angle equiprobable and then it interacts with tissue according to the tissue's optical properties at the photon's wavelength. Consequently, the deviation from the initial direction for fluorescent photons is less than for diffusely scattered, so the optical probing depth is also smaller.

As was expected, the fluorescent $g$-factor for $532 \mathrm{~nm}$ excitation is smaller than that for $632.8 \mathrm{~nm}$ excitation. It was mentioned before that effective fluorescent $g$-factor depends on the excitation wavelength and further propagation of fluorescent photons. After a fluorescent photon emission, process of diffusion is the same, and obtained distribution depends only on the path which excitation photon travelled. Emitted photons of a longer wavelength propagate further into biotissue than photons at a shorter wavelength, so excitation of a fluorophore and the following emission of photons occur deeper than at a longer wavelength excitation. As a result, the effective fluorescent $g$-factor is closer to 1 at a longer excitation wavelength. 


\section{Conclusion}

The results obtained are applicable for the FD of brain tissue where precise knowledge about spectral data obtained by means of FD is required. The use of longer wavelengths, as well as the use of a wider distance between source and detector, could possibly enable deeper tissue regions to be reached. This can be seen in the experiment, where the optical probing depth of the emission irradiation with the $532 \mathrm{~nm}$ wavelength was found to be $20-30 \%$ less than for the $632.8 \mathrm{~nm}$ wavelength. The most probable path of photons can be determined from the DT and MC simulations. Our calculations showed that both of these two methods correspond well with the experiment described in this paper. The middle region or the optical probing depth matches each of the methods, unless there is a difference near the fibre ends. Furthermore, the optical probing depth is proportional to the logarithmic function which depends on the distance between source and detector with the $R^{2}$ equal to 0.95 for the $632.8 \mathrm{~nm}$ and to 0.98 for the $532 \mathrm{~nm}$ wavelengths. Even though the choice of the excitation wavelength depends on the type of photosensitiser, it is also recommended to take the difference in the probing depth between excitation and fluorescent photons into account. This is due to the $60-70 \%$ difference in the probing depth (in the investigated wavelengths region), causing collection of the fluorescent photons from the regions lying above the area of the excitation photons path.

\section{Conflict of Interests}

The authors declare that there is no conflict of interests regarding the publication of this paper.

\section{References}

[1] J. de Leeuw, H. C. de Vijlder, P. Bjerring, and H. A. M. Neumann, "Liposomes in dermatology today," Journal of the European Academy of Dermatology and Venereology, vol. 23, no. 5, pp. 505-516, 2009.

[2] N. Fotinos, M. A. Campo, F. Popowycz, R. Gurny, and N. Lange, "5-Aminolevulinic acid derivatives in photomedicine: characteristics, application and perspectives," Photochemistry and Photobiology, vol. 82, no. 4, pp. 994-1015, 2006.

[3] Q. Peng, T. Warloe, K. Berg et al., "5-Aminolevulinic acid-based photodynamic therapy. Clinical research and future challenges," Cancer, vol. 79, no. 12, pp. 2282-2308, 1997.

[4] R. Richards-Kortum and E. Sevick-Muraca, "Quantitative optical spectroscopy for tissue diagnosis," Annual Review of Physical Chemistry, vol. 47, pp. 555-606, 1996.

[5] V. Tuchin, Tissue Optics: Light Scattering Methods and Instruments for Medical Diagnosis, SPIE Press, Bellingham, Wash, USA, 2000.

[6] V. B. Loschenov, V. I. Konov, and A. M. Prokhorov, "Photodynamic therapy and fluorescence diagnostics," Laser Physics, vol. 10, no. 6, pp. 1188-1207, 2000.

[7] A. A. Stratonnikov and V. B. Loschenov, "Evaluation of blood oxygen saturation in vivo from diffuse reflectance spectra," Journal of Biomedical Optics, vol. 6, no. 4, pp. 457-467, 2001.
[8] M. S. Patterson, B. Chance, and B. C. Wilson, "Time resolved reflectance and transmittance for the non-invasive measurement of tissue optical properties," Applied Optics, vol. 28, no. 12, pp. 2331-2336, 1989.

[9] S. Feng, F. A. Zeng, and B. Chance, "Photon migration in the presence of a single defect: a perturbation analysis," Applied Optics, vol. 34, no. 19, pp. 3826-3837, 1995.

[10] W. Cui, C. Kumar, and B. Chance, "Experimental study of migration depth for the photons measured at sample surface," in Time-Resolved Spectroscopy and Imaging of Tissues, vol. 1431 of Proceedings of SPIE, pp. 180-191, Los Angeles, Calif, USA, January 1991.

[11] S. A. Prahl, M. Keijzer, S. L. Jacques, and A. J. Welch, "A Monte Carlo model of light propagation in tissue," in Dosimetry of Laser Radiation in Medicine and Biology, vol. 5 of Proceedings of SPIE, pp. 102-111, 1989, http://omlc.ogi.edu/ prahl/pubs/abs/ prahl89.html.

[12] R. C. Gonzalez and R. E. Woods, Digital Image Processing, Person Prentice Hall, Upper Saddle River, NJ, USA, 3rd edition, 2007.

[13] R. Michels, F. Foschum, and A. Kienle, "Optical properties of fat emulsions," Optics Express, vol. 16, no. 8, pp. 5907-5925, 2008.

[14] S. T. Flock, S. L. Jacques, B. C. Wilson, W. M. Star, and M. J. C. van Gemert, "Optical properties of intralipid: a phantom medium for light propagation studies," Lasers in Surgery and Medicine, vol. 12, no. 5, pp. 510-519, 1992.

[15] A. N. Yaroslavsky, P. C. Schulze, I. V. Yaroslavsky, R. Schober, F. Ulrich, and H.-J. Schwarzmaier, "Optical properties of selected native and coagulated human brain tissues in vitro in the visible and near infrared spectral range," Physics in Medicine and Biology, vol. 47, no. 12, pp. 2059-2073, 2002.

[16] W.-F. Cheong, S. A. Prahl, and A. J. Welch, "A review of the optical properties of biological tissues," IEEE Journal of Quantum Electronics, vol. 26, no. 12, pp. 2166-2185, 1990. 

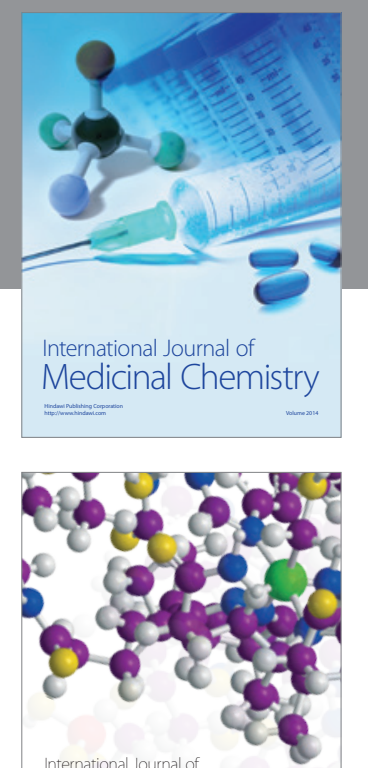

\section{Carbohydrate} Chemistry

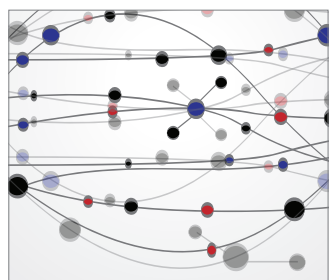

The Scientific World Journal
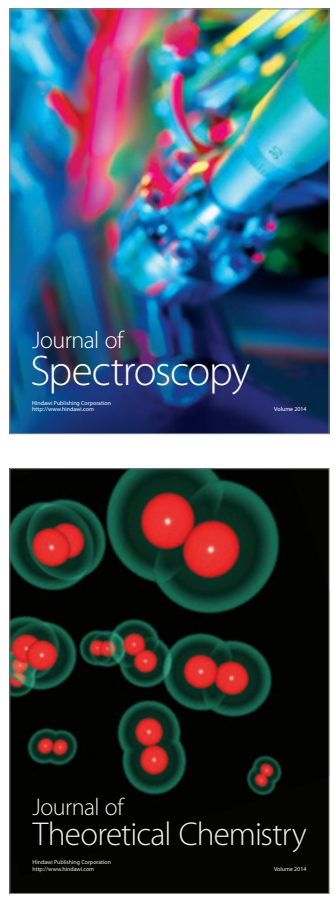
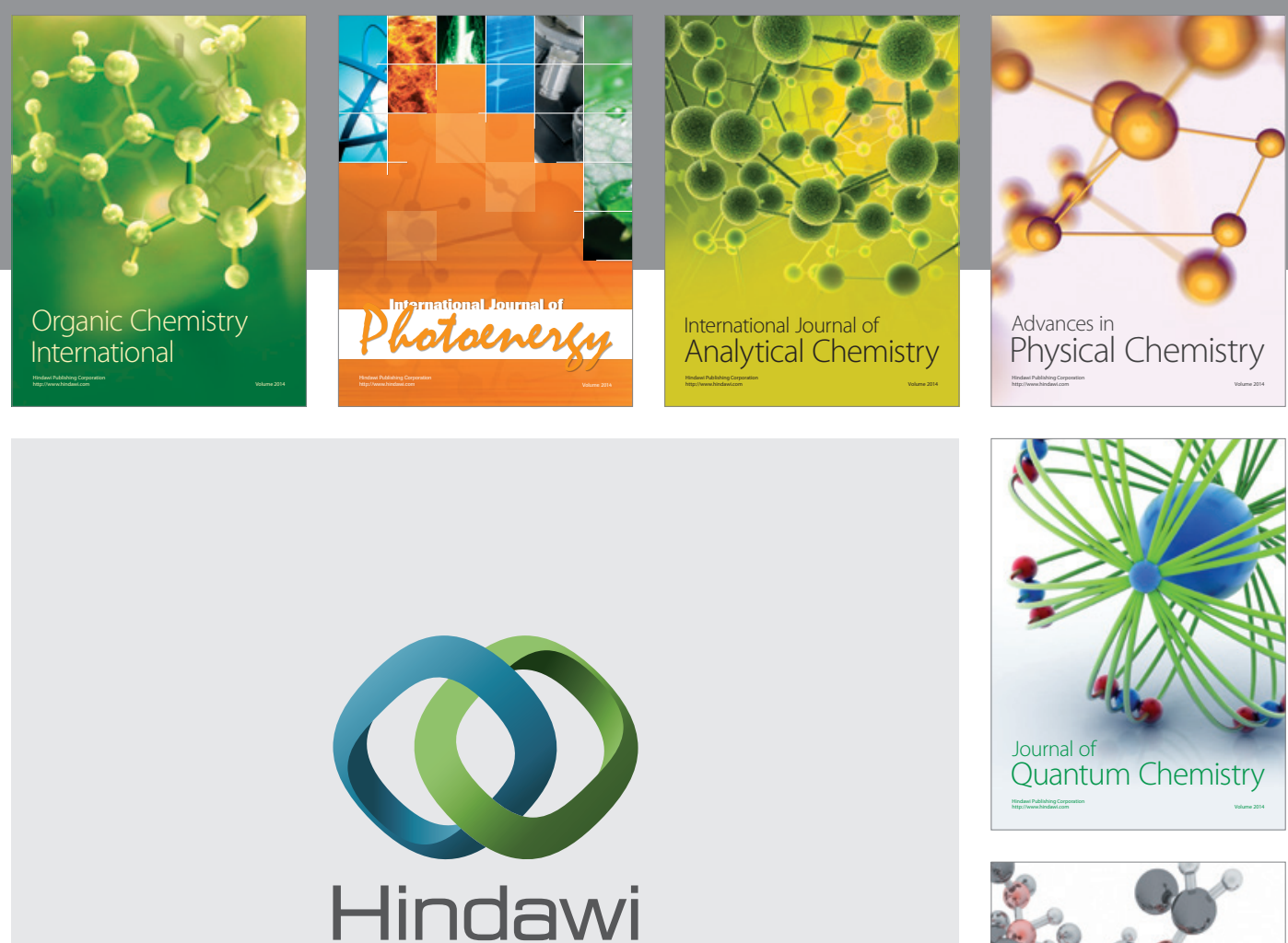

Submit your manuscripts at

http://www.hindawi.com

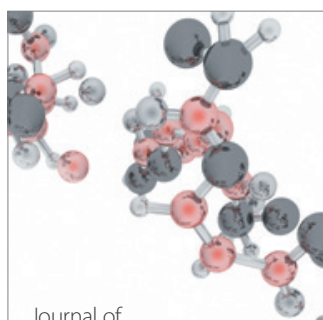

Analytical Methods

in Chemistry

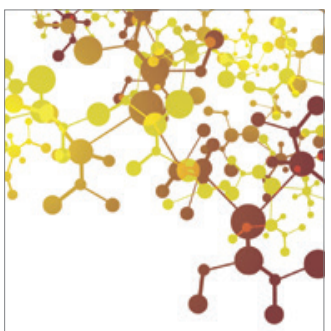

Journal of

Applied Chemistry

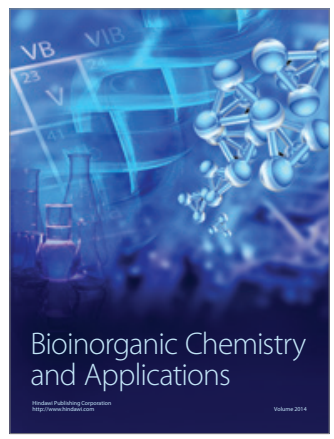

Inorganic Chemistry
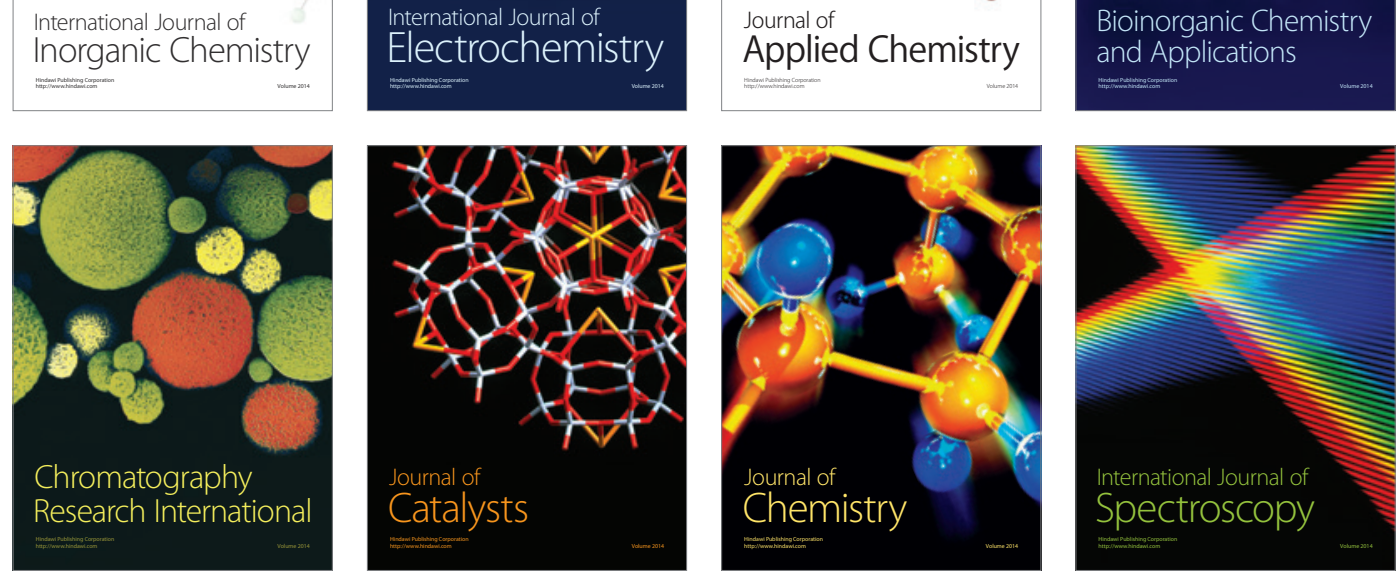